\title{
Development of communication skills in healthcare: Perspectives of new graduates of undergraduate nursing education
}

\author{
Lisa Kennedy Sheldon ${ }^{* 1,2}$, Dany M. Hilaire ${ }^{1,3}$ \\ ${ }^{1}$ College of Nursing and Health Sciences, University of Massachusetts Boston, Boston, United States \\ ${ }^{2}$ The Cancer Center, St. Joseph Hospital, Nashua, United States \\ ${ }^{3}$ Brigham and Women's Hospital, Boston, United States
}

Received: February 3, 2015

DOI: $10.5430 /$ jnep.v5n7p30
Accepted: April 14, 2015

Online Published: April 27, 2015

\begin{abstract}
Background: Communication between healthcare providers and patients affects patient care and safety. Nurses develop communication skills at the undergraduate level, after graduation and throughout their practice. This study explored perspectives of new graduates on the development and implementation of communication skills within the first six months after graduation.

Methods: Descriptive study using an electronic survey to three cohorts of recent graduates. Surveys were sent within six months after graduation.

Results: A total of 700 surveys were sent to three cohorts of recent graduates with 206 surveys completed (response rates ranged from 26\%-35\%). Participants reported always feeling confident communicating with patients and families $27 \%$ of the time, and $23.5 \%$ of the time with interdisciplinary teams. Only $50.8 \%$ reported always feeling confident to provide safe care all of the time with $44.5 \%$ reported feeling always able to ask colleagues for help with challenging situations.

Conclusions: Undergraduate nursing programs should incorporate more didactic communication skills training and simulation to prepare nursing students and increase their confidence to provide safe care and consult with colleagues for help. Communication skills training in practice settings after graduation to increase skill development and confidence during independent clinical practice.
\end{abstract}

Key Words: Communication, Nurses, Education, Safety, Cultural awareness

\section{INTRODUCTION}

Communication between health care providers and patients affects patient outcomes. Safe practice depends on effective communication. The Quality and Safety Education for Nurses (QSEN) links communication knowledge, attitudes and skills to improved patient outcomes and quality of care. ${ }^{[1]}$ Communication skills enable effective interactions between nurses and patients, and nurses and other healthcare workers. ${ }^{[2]}$ Improving communication among health care providers has been identified as part of the 2015 National Safety Goals. ${ }^{[3]}$ Furthermore, the goals and objectives of Healthy People 2020 recognize the importance of effective communication between health care providers and patients to improve patient outcomes and decrease adverse events. ${ }^{[4]}$

Healthcare providers, including nurses, begin developing pro-

\footnotetext{
${ }^{*}$ Correspondence: Lisa Kennedy Sheldon; Email: lisa.kennedysheldon@umb.edu; Address: College of Nursing and Health Sciences, University of Massachusetts Boston, 100 Morrissey Boulevard, Boston, United States.
} 
fessional communication skills during pre-professional education. In nursing, undergraduate didactic lectures, simulations, classroom interactions and clinical experiences provide a foundation for the later development of the professional communication skills used during independent clinical practice. ${ }^{[5]}$ After graduation and registration or licensure, nurses further develop and refine their communication skills as independent professionals working within healthcare teams in unique settings. Developing these skills is an ongoing process as newly graduated nurses integrate undergraduate preparation into experiences during clinical practice. Understanding the perspectives of newly graduated nurses during the first six months after graduation informs undergraduate teaching pedagogies and continuing education to prepare nurses as skilled, confident communicators with patients, families, and other healthcare team members. Thus the purpose of this study was to describe the perception of newly graduated nurses regarding their communication skills in a variety of common clinical situations.

\subsection{Literature review}

Communication skills are an essential component of health care. Effective communication skills are even more important for nurses as they are at the forefront of patient care providing $80 \%$ of healthcare services globally. ${ }^{[6]}$ The landmark Robert Wood Johnson Foundation Carnegie report entitled, Educating nurses: A call for radical transformation highlighted the importance of incorporating communication skills training into nursing education across the curriculum. ${ }^{[7]}$ In addition to the inclusion of the didactic training of communication skills, Benner and colleagues called for including more training and role-playing in nursing programs to promote the development of effective communication skills. ${ }^{[7]}$

A variety of pedagogies have been employed in communication skills training for undergraduate nursing students including lectures, role-play, case studies, and simulations. ${ }^{[8,9]}$ Zavertnik and colleagues (2010) assessed the effectiveness of a learner-centered simulation intervention to improve the communication skills of sophomore (second year) nursing students. This pilot study used a two group, post-test design with 21 nursing students. The intervention group received a formal teaching session which included a framework for communication and guidelines developed by Lorin et al. (2006) and the control group received no special teaching session. ${ }^{[10]}$ This framework focused on four communication domains; introduction, gathering of information, imparting information, and clarifying goals. ${ }^{[10]}$ Zavertnik and colleagues (2010) assessed these domains during individual testing sessions with an actor posing as a family member. ${ }^{[8]}$ Nurse educators, blinded to whether students received the

Published by Sciedu Press intervention, evaluated the students' communication skills during a clinical simulation. Students in the intervention group performed more effectively in all four domains when compared to the control group and were significantly more effective in gathering information $(p=.0257)$. In addition, those in the intervention group were more effective at asking open-ended questions, listening effectively, and discussing important information. ${ }^{[8]}$

Xie and colleagues (2012) investigated nursing students' communication skills at the beginning of first clinical practicum experience. ${ }^{[11]}$ Students from 22 universities in China ( $n=322$ ) were given four questionnaires to self-assess their communication skills. The questionnaires collected demographic data, and assessed students' perceptions of their overall communications skills before they started clinical practicum sessions. Interestingly, students who had completed communication skills training reported better communication skills. The majority of the nursing students (88.1\%) reported needing more education about communication skills prior to beginning clinical experiences. ${ }^{[11]}$ The authors advocated for communication content to be consistently included in the curricula.

When students communicate with patients from cultures different from their own, they may lack confidence and perceive more difficulty with their communication skills. Jirwe et al. (2010) explored student nurses' experiences of communication in cross-cultural care encounters. ${ }^{[12]}$ They conducted semi-structured interviews with 10 Swedish nursing students with Swedish or immigrant ethnic backgrounds. One of the themes supported the assumption that students did not feel comfortable caring for patients who spoke a language different from their own. Specifically, the students described communicating the technical aspects of care but they were not able to convey the more caring components of their communication. The students were reluctant to ask questions and concerned they may make mistakes when communicating with patients who spoke another language. Furthermore, the students' cultural knowledge or lack thereof impacted their communication with patients from different cultures. The students observed that nurses often did not use interpreters because of financial concerns. And although students acknowledged the benefit of using an interpreter, only one student had such an experience. As a result, they described other, less effective strategies such as using family as interpreters or gestures or hand movements to communicate with patients. ${ }^{[12]}$ In contrast, healthcare institutions in the U.S. are required to have professional translators available to facilitate communication, avoid miscommunication and promote safer care. ${ }^{[13]}$ 
In summary, studies regarding communication skills training for nursing students have assessed the type of training, the role of simulation, and the integration of communication skills training from other disciplines. ${ }^{[8,9]}$ Students have been evaluated with post- tests to evaluate perceptions of their own communication skills. ${ }^{[11]}$ However, to our knowledge, there are no studies to date that have assessed the perception of new nurse graduates regarding their communication skills in independent clinical practice. The results of this study will inform the need for a broader communication curriculum in undergraduate nursing programs and the needs of newly graduated nurses during the transition from new graduate to independent clinician.

\subsection{Conceptual framework}

The conceptual framework guiding this study is Benner's Novice to Expert Model (1984). ${ }^{[14]}$ Benner's Model describes the development of both skills and understanding in patient care over time after an educational foundation. It further states that experience is a prerequisite of becoming an expert, not just acquisition of knowledge. The model identifies five levels of competence for nurses: novice, advanced beginner, competent, proficient, and expert. The novice has no experience and lacks confidence to demonstrate safe practice. The advanced beginner demonstrates acceptable performance, requiring occasional supportive cues as knowledge is developing. The advanced beginner also recognizes a critical situation but may require guidance to understand and anticipate interventions or the necessary care. The competent nurse demonstrates efficiency and has confidence in his or her actions. The proficient nurse perceives situations as a whole and thinks about long-term goals. Lastly, the expert nurse has an intuitive grasp on all situations and is able to understand the small details of a problem and adapt care to the individual patient. ${ }^{[14]}$ Applying Benner's Model to the acquisition of communication skilss, it is expected that communication skills will improve as a nurse moves through the different levels of competence, specifically during their initial transition after completing undergraduate education.

\section{Methods}

This descriptive study used mixed methods to explore the perceptions of newly graduated nurses regarding their communication skills within six months of graduation. The survey items were developed in an iterative process using a team of nurse educators and an expert on nurse communication. The team reviewed literature reviews and clinical scenarios over six meetings. New graduate perspectives on the survey items were gathered in face-to-face three interviews and discussed with the team. The survey for the first cohort consisted of fourteen Likert-type questions and seven open- ended questions with text box entries. Likert-type responses were always, most of the time, sometimes, rarely and never.

The survey was modified after the first cohort to decrease respondent burden and increase the response rates. Four of the open-ended text box responses were deleted due to redundancy of information in other items and to decrease response time and still capture the content. The modification also decreased the total number of Likert-type scale questions from fourteen to seven due to redundancy of content. The seven Likert-type questions in the modified survey explored graduates perceptions about their: (1) confidence communicating with patients, families; (2) confidence communicating within interprofessional teams; (3) satisfaction with their communication skills; (4) ability to ask colleagues for help; (5) comfort assessing health literacy; (6) competence providing safe care; and (7) preparation to integrate cultural awareness in practice. Likert-type responses were always, most of the time, sometimes, rarely and never. The three open-ended questions in the modified survey asked graduates to describe: 1) an incident when they resolved a conflict; 2) a situation where they felt that gained confidence in communication skills; and 3) an incident that challenged their communication skills. The final survey included demographic information, seven Likert-type questions, and three open-ended questions.

An online survey was used to increase participation from newly graduated nurses who may have been working as a nurse in a new job or in more distant geographical locations from the university. The sample consisted of three cohorts of newly graduated nurses from a large, urban, public university in the Northeast United States. The sample included graduates from two baccalaureate nursing options: 1) full time undergraduate program; and 2) second-degree, undergraduate accelerated program. Students were invited to participate in the study via email after their graduation.

\subsection{Data collection}

The Institutional Review Board (IRB) at the university approved the study (UMB IRB 20100921) and determined the study had exempt status meaning that participation provided minimal risk and completion of the online survey indicated consent to participate. Electronic online surveys (using Survey Monkey $\left.{ }^{\mathrm{R}}, 1999-2014\right)^{[15]}$ were sent to three different cohorts of recent graduates between 2009 and 2012 (see Table 1). The survey was sent to the university email addresses of newly graduated nurses within six months after graduation. Graduates had one month to complete the survey. Weekly reminders were sent via email. Graduates had the option to not complete the survey and also to withdraw from receiving further emails regarding the surveys. Respondents were 
given a token gift card after completion of the survey.

\subsection{Data analysis}

Data were analyzed using descriptive statistics including means and percentages, to describe and synthesize the quantitative data from the demographic questions. All data were analyzed using STATA 12.0 (Stata Corporation, 2011). ${ }^{[16]}$ The data from the items on the first survey were merged with data from the same items on the modified survey used for the second and third cohorts. Only items used on all three surveys were analyzed. Items which were dropped from the first survey (i.e., four open ended questions and seven Likert-type questions) were omitted from the analyses. Cronbach alphas for the three surveys ranged from .69 on the first survey to .71 for the second and third surveys. Summative content analyses were used to review text box responses to the open-ended questions. Those replies were reviewed for common themes. ${ }^{[17]}$

\section{RESULTS}

A total of 700 surveys were sent to newly graduated nurses with 206 respondents. Respondents' ages ranged from 22 to 60 years with a mean of $30.2(\mathrm{SD}=9.4)$ and a median age of 27. Eighty-three percent $(n=171)$ of respondents were female. Over a third $(37.3 \%)(n=75)$ of the respondents represented a minority. Nearly half (49\%) of the respondents had previously earned undergraduate degrees other than nursing degrees (see Table 1). Seventy percent of respondents were currently employed as nurses. The overall response rate to the survey invitation was $31 \%$ but varied by cohort and ranged from $26 \%$ to $35 \%$ (see Table 1 ). The response rate for the first cohort was $26 \%$ and prompted the survey modification with reduction in Likert-type questions from 14 to seven and a decrease in open-ended text box responses from seven to three items. The cohorts did not vary significantly by age, gender or educational programs (traditional versus accelerated) across the three cohorts.

Table 1. Demographic data and response rates $(\mathrm{N}=206)$

\begin{tabular}{|c|c|c|c|c|}
\hline & $\begin{array}{l}\text { Overall } \\
n=206\end{array}$ & $\begin{array}{l}\text { Cohort } 1 \\
n=46\end{array}$ & $\begin{array}{l}\text { Cohort } 2 \\
n=58\end{array}$ & $\begin{array}{l}\text { Cohort } 3 \\
n=102\end{array}$ \\
\hline \multicolumn{5}{|l|}{ Age (years) } \\
\hline Range & $22-60$ & $22-53$ & $22-46$ & $22-60$ \\
\hline Mean & 30.2 & 29.7 & 28.2 & 32.4 \\
\hline Median & 27 & 25 & 26 & 29 \\
\hline SD & 9.4 & 9.0 & 9.1 & 9.2 \\
\hline \multicolumn{5}{|l|}{ Gender } \\
\hline Female & $171(83 \%)$ & 34 (74\%) & $53(86.2 \%)$ & $84(83.2 \%)$ \\
\hline Male & 35 (17\%) & 12 (26\%) & $8(13.8 \%)$ & 15 (16.8\%) \\
\hline \multicolumn{5}{|l|}{ Marital Status } \\
\hline Single & 127 (62.6\%) & $35(76 \%)$ & 39 (67.2\%) & $59(57.4 \%)$ \\
\hline Married & 68 (33\%) & $11(24 \%)$ & $16(27.6 \%)$ & $38(37.2 \%)$ \\
\hline Separated & $2(1 \%)$ & 0 & $1(\%)$ & $1(1 \%)$ \\
\hline Divorced & $3(1.4 \%)$ & 0 & $1(\%)$ & $2(2 \%)$ \\
\hline Partnered & $6(3 \%)$ & 0 & $3(\%)$ & $3(2.4 \%)$ \\
\hline \multicolumn{5}{|l|}{ Culture/ethnicity } \\
\hline Caucasian/non-Hispanic & 135 (\%) & $32(70 \%)$ & 37 (64.8\%) & $68(66.7 \%)$ \\
\hline Hispanic & $9(4.4 \%)$ & 0 & $3(5.2 \%)$ & $5(4.9 \%)$ \\
\hline African American/Black & $32(15.5 \%)$ & $8(17 \%)$ & $8(13.8)$ & $16(15.7 \%)$ \\
\hline Asian/Pacific Islander & $26(12.6 \%)$ & $8(17 \%)$ & $10(17.2 \%)$ & $12(11.8 \%)$ \\
\hline Native American & $1(0.4 \%)$ & 0 & 0 & $1(1 \%)$ \\
\hline Other & $7(4.4 \%)$ & $1(2 \%)$ & 0 & $1(1 \%)$ \\
\hline \multicolumn{5}{|l|}{ Previous Degrees } \\
\hline Yes, other than nursing & $101(49 \%)$ & $16(35 \%)$ & 22 (38\%) & $55(54 \%)$ \\
\hline No & 105 (51\%) & $30(65 \%)$ & $36(62 \%)$ & 47 (46\%) \\
\hline \multicolumn{5}{|l|}{ Response Rates } \\
\hline Sent & 600 & 130 & $185^{*}$ & $385^{*}$ \\
\hline Returned & 206 & 46 & 58 & 102 \\
\hline Response Rate & $31 \%$ & $26 \%$ & $31 \%$ & $36 \%$ \\
\hline Cronbach alpha & & 0.69 & 0.71 & 0.71 \\
\hline
\end{tabular}

*Modified survey 


\subsection{Confidence with communication skills: Six months} after graduation

Six months after graduation, $44.5 \%(n=85)$ of recent graduates reported that they always felt confident asking colleagues for help and 50.8\% always felt competent to provide safe care $(n=97)$. Recent graduates reported to feel confident most of the time communicating with patients and families $(59.7 \%)$ and communicating with interprofessional teams $(50.3 \%)$. They were satisfied most of the time with their communication skills $(56.5 \%)$. Only $27 \%(\mathrm{n}=53)$ of respondents reported feeling always confident communicating with patients and families. Forty-five respondents (23.5\%) reported to always feeling confident communicating within interdisciplinary teams, and only $22.5 \%$ reported to always feel satisfied with their communication skills. When asked about confidence assessing health literacy, half of respondents $(50.8 \%)$ reported being confident most of the time. Eight-four percent $(n=156)$ of recent graduates reported that they felt always or most of the time prepared to integrate cultural awareness and sensitivity into their practice (see Table 2).

Table 2. Perspectives of newly graduated nurses on communication skills $(\mathrm{n}=191)^{*}$

\begin{tabular}{|c|c|c|c|c|c|}
\hline How often do you feel: & Always & Most of the time & Sometimes & Rarely & Never \\
\hline Confident communicating with patients and families & $27 \%(53)$ & $59.7 \%(114)$ & $11.5 \%(22)$ & $1 \%(2)$ & 0 \\
\hline Confident communicating with interdisciplinary teams & $23.5 \%(45)$ & $50.3 \%(96)$ & $19.9 \%(38)$ & $2.6 \%(5)$ & 0 \\
\hline Satisfied with communication skills & $22.5 \%(43)$ & $56.5 \%(108)$ & $20.4 \%(39)$ & $2.1 \%(4)$ & 0 \\
\hline $\begin{array}{l}\text { Able to ask colleagues for help with challenging } \\
\text { situations }\end{array}$ & $44.5 \%(85)$ & $41.9 \%(80)$ & $11 \%(21)$ & $1.6 \%(3)$ & 0 \\
\hline Comfortable assessing patient health literacy & $30.9 \%(59)$ & $50.8 \%(97)$ & $15.7 \%(30)$ & $1.6 \%(3)$ & 0 \\
\hline Competent to provide safe care & $50.8 \%(97)$ & $44 \%(84)$ & $3.1 \%(6)$ & 0 & 0 \\
\hline $\begin{array}{l}\text { Prepared to integrate cultural awareness/sensitivity into } \\
\text { practice }\end{array}$ & $39.8 \%(76)$ & $44.6 \%(85)$ & $13.6 \%(26)$ & 0 & 0 \\
\hline
\end{tabular}

$*_{n}=191$ of the 206 respondents completed the Likert-type questions.

\subsection{Open-ended questions}

Both the initial survey and the modified survey included three open-ended questions asking respondents to: 1) describe a situation where they played a role in resolving a conflict; 2) describe a situation when they gained confidence in communication skills; and 3) describe a situation that challenged their communication skills. Forty percent $(n=$ 93) of the respondents completed the open-ended questions. Several topics emerged during content analyses of the text box responses to the three questions.

\subsubsection{Successfully resolving a conflict}

Respondents identified two types of situations where they played a role in successfully resolving a conflict: with coworkers $(\mathrm{n}=93)$, and with patients and/or their families $(n=79)$. For example, one respondent described a patient situation, writing: "A patient's family had been given two different sets of information regarding a patient's discharge plans so I went to the chart and referred to case management notes to come up with a resolution". In this scenario, the respondent described clarifying the information given to the patient to lessen his/her anxiety prior to discharge. For an example of resolving a conflict with a co-worker(s), one respondent described his/her role of intervening between two physicians. As described by the respondent, "De-escalated a 34 miscommunication between two doctors to remind them that the patient is what was most important at the moment".

\subsubsection{Gained confidence in communication skills}

When asked to "describe a situation where you gained confidence in communication skills", three themes emerged from the respondents: 1) communication with higher-level personnel; 2) a process of becoming more confident communicating with patients or patients' families; and 3) communication with co-workers. The first theme, communication with higher-level personnel, emerged in multiple responses. Respondents described the roles of confidence and communication with colleagues in higher-levels (e.g., managers) and other colleagues (e.g., doctors). One respondent wrote, "In codes, I gain confidence in my ability to communicate with all members of the healthcare team". Another respondent commented, "Discussing patient's needs and issues with physicians and other providers help me gain confidence in my communication skills". Both of these descriptions emphasize the positive influence of colleagues in helping newly licensed nurses successfully advocate for patients and thereby gain confidence in interprofessional communication.

The second theme was the process of becoming more confident communicating with patients or patients' families. One respondent wrote: "This (confidence) is something that hap- 
pens slowly with each interaction with patients. Each small communication makes the next interaction easier until you feel comfortable speaking about a wide range of topics". Lastly, the third theme revolved around communication with co-workers. One respondent described positive interactions during undergraduate clinical rotations that increased confidence. The respondent stated: "During debriefing with my clinical instructors after each shift helped me with my communication skills. We always discussed what could have gone better and ideas for improvement".

\subsubsection{Challenges in communication skills}

The third open-ended question asked respondents to "describe a situation that challenged your communication skills". Three themes emerged during the content analysis: 1) lack of confidence; 2) language barriers; and 3) horizontal or lateral violence. The first theme, lack of confidence, was described as the effect of challenging situations that affect communication with patients and patients' families. Respondents did not feel confident in answering questions from patients and families. One respondent shared: "I feel rushed all the time and forget about tasks I agreed to do for patients or colleagues. Also I don't feel confident personally which affects my advocacy skills for patients". The second theme, language barriers, occurred when patients did not understand or speak English. For example a respondent wrote, "Having patients that speak little to no English is always a challenge, especially when there is no interpreter available". Another respondent gave another example, "I had a challenging situation when I had to do discharge teaching on a patient whom did not speak English". Lastly, a theme of horizontal or lateral violence was described as a challenging situation when conflicts occurred or when a respondent felt intimated by co-workers. One respondent shared an example of lateral violence:

"As a new R.N., I worked one night with another new R.N., a more senior R.N., and a nurse leader. The two experienced nurses were far short of helpful with anything, particularly two specific patients in dangerous conditions. Both myself and the other new R.N. were challenged in finding ways to report the lack of support and leadership we received that night. We asked for help and did not receive it. We struggled in professionally communicating the situation to our manager."

The respondent's perceived intimidation by the experienced nurses hindered this newly graduated nurse from speaking to the manager about the issue, a situation described as one which affected patient safety. Another respondent described a similar challenge "My communication skills have been challenged in the past when communicating with surgeons who can come off as intimidating, short in their responses, and quick to judge negatively".

\section{Discussion}

The findings in this study supported Benner's theory that the development of confidence and the application of communication skills are developmental processes occurring with independent practice and repetition. ${ }^{[14]}$ New nurses in our study were comfortable assessing health literacy $(81.7 \%)$ and prepared to integrate cultural awareness and sensitivity into practice $(84.4 \%)$, in contrast to the findings of Jirwe and colleagues (2010) reporting nursing students lacked skills and confidence in cross-cultural communication and encounters. ${ }^{[12]}$ However, language barriers with patients was a common finding between the students surveyed by Jirwe and colleagues (2010) and the respondents to this survey who described language barriers as one of the most challenging communication scenarios. ${ }^{[12]}$

In our study, nurse respondents reported experiences that strengthened their communication skills with patients and their families, and with colleagues in interprofessional healthcare teams. Overall, a majority of newly graduated nurses felt confident most of the time about their communication skills. However, they only reported feeling always confident to provide safe care half of the time $(50.8 \%)$ and able to ask for help from colleagues $44.5 \%$ of the time. These are discouraging findings because while safety is stressed in undergraduate education half of the newly-graduated nurses did not feel confident to provide safe care nor ask for help from colleagues. These findings have significant implications for patient safety and may be attributable to lack of mentorship from colleagues, shortened orientation programs for new graduates, or hostility in the workplace ${ }^{[18,19]}$ or their increasing sense of responsibility as newly licensed professionals.

As described by the respondents' comments, coworkers had an increasingly important effect on helping new nurses gain confidence. There are known challenges of communicating within the triad of nurse-doctor-patient interactions and these have been identified as one of the most difficult communication scenarios by nurses. ${ }^{[20]}$ The respondents identified specific examples when interactions and communication with coworkers including higher-level personnel, both increased their confidence or challenged their communication skills. This finding supports Benner's description of professional development ${ }^{[14]}$ and the need for continuous mentorship of newer graduates to improve their communication skills and improve the safety of patient care.

Different scenarios and incidences may also help new graduates identify areas where they need to improve of their communication skills or ask for more training when they 
doubt their ability to respond in novel situations. Six months after graduation the responses of the graduates support the practice of a novice nurse, demonstrating rule-governed behavior. $^{[14]}$ In Benner's next stage, advanced beginner, new nurses would demonstrate increased in knowledge from experiences to guide actions, and ultimately would identify where they needed more knowledge and support.

New graduates gave descriptive examples of the three scenarios proposed in the open-ended questions: 1) the successful resolution of a conflict; 2) an incident where they gained confidence in communicating; and 3) an incident that challenged their communication skills. The themes that emerged from the responses included incidents where new graduates worked with patients and patient families as well as incidents with co-workers. In congruence with the findings of Jirwe et al. (2010), language barriers were overwhelmingly the most challenging situation for new graduates. In our study, new nurse graduates faced similar challenge of working with patients with language barriers. A study by Kale \& Syed in which over $50 \%$ of the respondents were nurses, further emphasized the difficulty accessing interpreter services for patients who do did not speak English as a first language. ${ }^{\text {[21] }}$ These findings point to the need for nursing curricula to provide education to prepare future nurses to work with patients and their families who speak different languages, respect diverse cultures and learn how to use and access resources such as interpreter services to overcome language barriers and improve the quality and safety of care.

\section{Conclusion}

Communication skills are essential in health care and strongly influence patient outcomes. The results of this study provide data regarding new graduate perspectives on the development of communication skills and confidence during early independent clinical practice. It is important that didactic, role-playing, simulation exercises as well as practical training in communication skills are incorporated with increasing complexity in all undergraduate nursing curricula. These exercises will increase students' skills and build their confidence prior to graduation and help prepare for independent clinical practice. In addition, it is vitally important that employers recognize the importance of extending orientation, mentorship programs, and debriefing sessions for newly graduated nurses to provide continued support and ensure patient safety and collegial mentorship. A mentor to a newly graduated nurse should be competent, proficient or expert, as defined by Benner's model (1984), indicating a nurse with at least three years of clinical experience. ${ }^{[14]}$ Furthermore, communication skills should also be included in the workplace for newly graduated nurses as they transition from student to independent clinician. In Benner's Model (1984), it is reported that it may take two years for newly graduated nurses to become competent in providing care. ${ }^{[14]}$ Furthermore, ongoing workplace educational programs are needed to support practicing nurses so they can effectively communicate during difficult situations such as critical incidents, episodes of lateral violence, addressing language barriers, and ineffective team communication. Finally, practicing nurses could likely benefit from educational programs to learn peer debriefing techniques. These programs could focus on building nurses' clinical reasoning and confidence during complex or challenging clinical situations.

While our sample reported being comfortable integrating cultural awareness into patient care, newly graduated nurses would likely benefit from more cultural awareness and sensitivity training as they encounter patients from different backgrounds especially when patients do not speak the same language as the nurse. Our findings indicate that more resources such as telephone or online interpreter services are likely needed to help nurses care for patients who speak different languages in a more timely manner. In addition, institutional orientation programs should include appropriate access and use of interpreter services, resources, and available tools to help nurses care for patients from different cultures or who speak different languages.

\subsection{Limitations}

There were several limitations in this study. We chose to use electronic surveys to reach recent nurse graduates who may have been geographically dispersed. However, the response rate to our first survey was lower than expected. To respond to the initial lower and incomplete response rate, we modified the survey for the second and third cohorts. Although the content was maintained, the modification (as described in the Methods section) may have affected the responses and the response rate did not increase as much as expected. New graduates were recruited from only one large, public university in an urban area and findings may differ for graduates from other public universities or smaller, private schools or in other countries. This survey did not ask respondents to identify the specific institution or setting in which they worked.

For example, newly graduated nurses working in a teaching hospital may have different experiences than those working in a community hospital or skilled nursing facility. Respondents were not asked the length of time they had been working as a registered nurse since graduating. The survey used self report methods and did not include specific measures of health literacy or cultural awareness. 


\subsection{Future research}

Future research should ask new graduates what they would recommend to improve undergraduate and postgraduate education on communication skills. Studies could incorporate specific scales to measure health literacy and cultural awareness. These scales would help identify specific components of cultural awareness and sensitivity, and health literacy that are amenable to the development of interventions at the undergraduate level and for continuing education for practicing nurses. Future studies should include additional demographic information about respondents' current work settings and duration of practice. Furthermore, institutional programs should be implemented that enforce interprofessional training programs in academic and practice settings. These programs should emphasize communication skills, collaboration, collegiality, and conflict resolution to improve patient care processes, safety, and outcomes.

\section{CONFLiCTS OF INTEREST Disclosure}

The authors declare that there is no conflict of interest statement.

\section{REFERENCES}

[1] Cronenwett L, Sherwood G, Barnsteiner J, et al. Quality and safety education for nurses. Nursing Outlook. 2007; 55: 122-131.

[2] Sully P, Nicol M. Essential Communication Skills for Nursing. London; Elsevier Mosby; 2005; 94-95. PMid:17524799 http: //dx.doi.org/10.1016/j.outlook.2007.02.006

[3] The Joint Commission. Hospital National Patient Safety Goals. 2015. Available from: http://www.jointcommission.org/assets/1 /6/2015_hap_npsg_er.pdf

[4] United States Department of United States Department of Health and Human Services, Healthy People 2020. Health Communication and Health Information Technology. 2011. Available from: http://www.healthypeople.gov/2020/topicsobjec tives2020/overview. aspx?topicid=18

[5] Krautscheid LC. Improving communication among healthcare providers: Preparing student nurses for practice. International Journal of Nursing Education. 2008; 5(1): 1-13. http://dx. doi .org/10. 2202/1548-923X. 1647

[6] Hughes F. Nurses at the forefront of innovation. Internal Nursing Review. 2006; 53(2): 94-101. PMid:16650027 http://dx.doi.o $\mathrm{rg} / 10.1111 / \mathrm{j} .1466-7657.2006 .00463 . \mathrm{x}$

[7] Benner P, Stuphen M, Leonard V, et al. Educating nurses: A call for radical transformation. The Carnegie Foundation for the Advancement of Teaching. San Francisco CA: Jossey-Bass; 2010.

[8] Zavertnik JE, Huff TA, Munro CL. Innovative approach to teaching communication skills to nursing students. Journal of Nursing Education. 2001; 49(2): 65- 71. PMid:19810670 http://dx.doi .org/1 $0.3928 / 01484834-20090918-06$

[9] Aebersold M, Tschannen D, Sculli G. Improving nursing students' communication skills using Crew Resource Management Strategies. Journal of Nursing Education. 2013; 52(3): 125-130. PMid:23380022 http://dx.doi.org/10.3928/01484834-20130205-01

[10] Lorin S, Rho L, Wisnivesky JO, et al. Improving medical student intensive care unit communication skills: A novel educational initiative using standardized family members. Critical Care Medicine.
2006; 34: 2386-2391. PMid:16791111 http://dx.doi.org/10. 1097/01.CCM.0000230239.04781. BD

[11] Xie J, Ding S, Wang C, et al. An evaluation of nursing students' communication ability during practical clinical training. Nurse Education Today. 2012; 33: 823-827. PMid:22417661 http://dx.doi.org $/ 10.1016 / j$.nedt .2012 .02 .011

[12] Jirwe M, Gerrish K, Emami A. Student nurses' experiences of communication in cross- cultural care encounters. Journal of Caring Sciences. 2010; 24: 436-444. PMid:20233352 http://dx.doi.o $\mathrm{rg} / 10.1111 / \mathrm{j} .1471-6712.2009 .00733 . \mathrm{x}$

[13] National Council of Interpreting in Health Care. National Standards of Practice for Interpreters in Healthcare. 2005.

[14] Benner P. From Novice to Expert, Excellence and Power in Clinical Nursing Practice. Menlo Park, CA: Addison; 1984.

[15] Survey Monkey. Palo Alto, California. 2014.

[16] STATA Corporation. STATA Statistical Software: Release 12. College Station, TX: StataCorp LP. 2011.

[17] Hsieh HF, Shannon SE. Three approaches to qualitative content analysis. Qualitative Health Research. 2005; 15(9): 1277-1288. PMid:16204405 http://dx.doi.org/10.1177/1049732305276687

[18] Thomas SP, Burk R. Junior nursing student's experiences of vertical violence during clinical rotations. Nursing Outlook. 2009; 57(4): 226-231. PMid:19631065 http://dx.doi.org/10.1016/j.out look.2008.08.004

[19] Roche M, Diers D, Duffield C, et al. Violence toward nurses, the work environment, and patient outcomes. Journal of Nursing Scholarship. 2010; 42(1): 13-22. PMid:20487182 http://dx.doi.org /10.1111/j.1547-5069.2009.01321.x

[20] Sheldon LK, Barrett R, Ellington L. Difficult communication in nursing. Journal of Nursing Scholarship. 2006; 38(2): 141-147. http://dx.doi.org/10.1111/j.1547-5069.2006.00091.x

[21] Kale E, Syed HR. Language barriers and the use of interpreters in the public health services. A questionnaire-based survey. Patient Education and Counseling. 2010; 81(2): 187-191. PMid:20542656 http://dx.doi.org/10.1016/j.pec. 2010.05.002 